\title{
Electrostatic Manifestation of Micro-Heterogeneous Solvation Structures in Deep-Eutectic Solvents: A Spectroscopic Approach
}

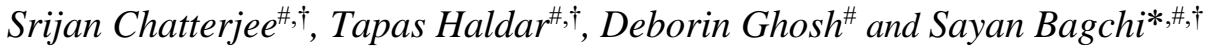 \\ \# Physical and Materials chemistry Division, CSIR-National Chemical Laboratory (CSIR- \\ NCL), Dr. Homi Bhabha Road, Pune 411008, India \\ $\dagger$ Academy of Scientific and Innovative Research (AcSIR), Ghaziabad - 201002, India \\ *s.bagchi@ncl.res.in, +91-20-25903048 (Tel),+91-20-25902636 (Fax)
}

To whom correspondence should be addressed. Email: s.bagchi@ncl.res.in. 
Figures:

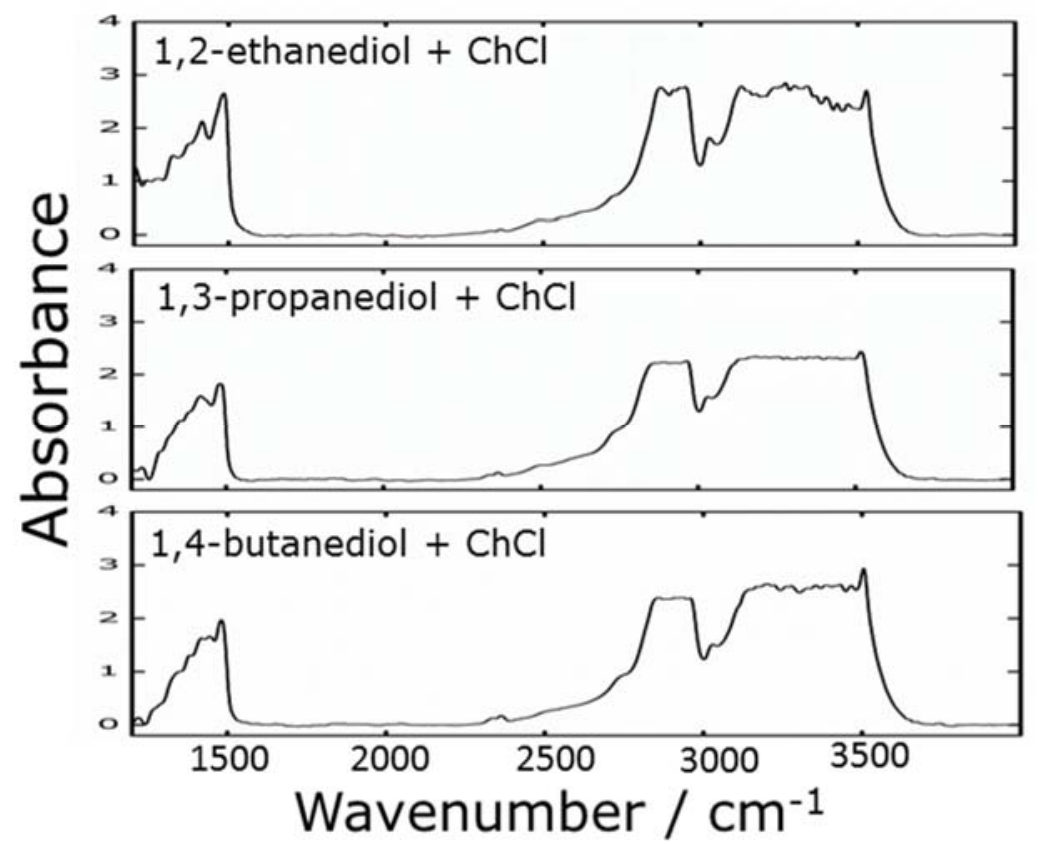

Figure S1. FTIR spectra of pure DESs

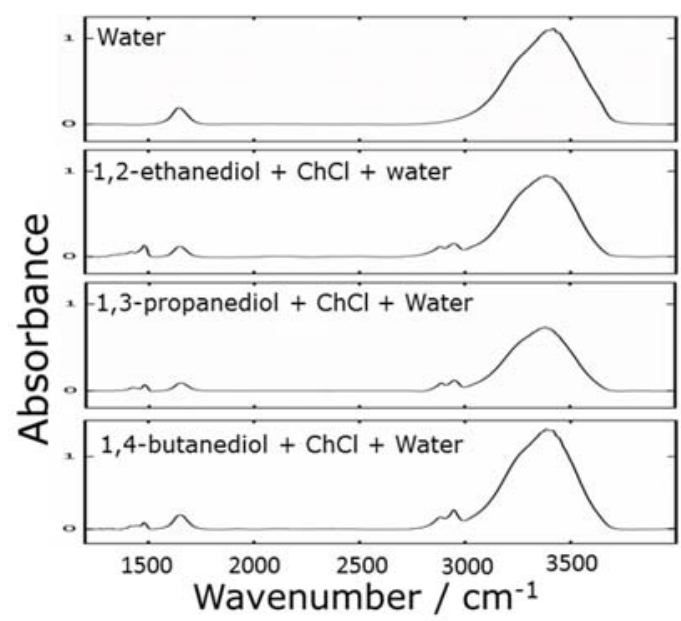

Figure S2. FTIR spectra of pure water and DES-water mixture $(2: 1 \mathrm{v} / \mathrm{v})$ 


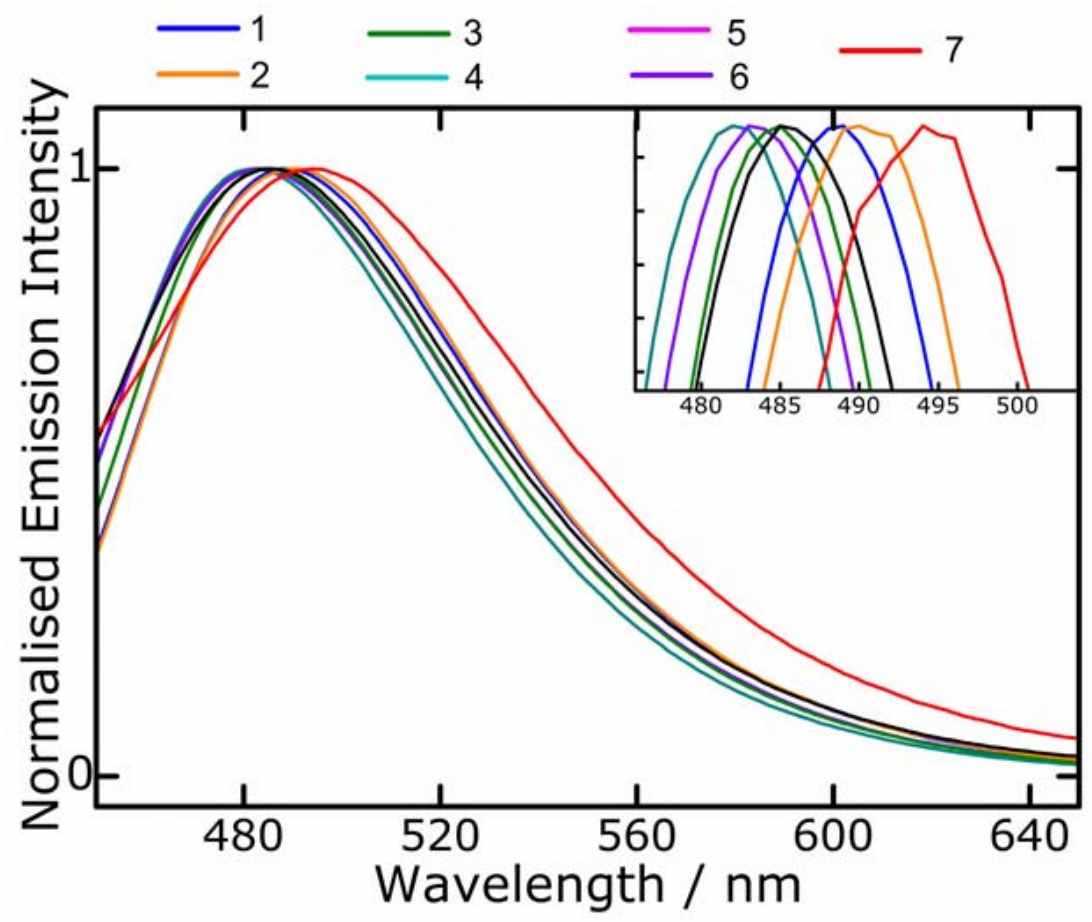

Figure S3. Emission spectra of coumarin151 $\left(\lambda_{\mathrm{ex}}=350 \mathrm{~nm}\right)$ in different deep eutectic solvents (DESs).

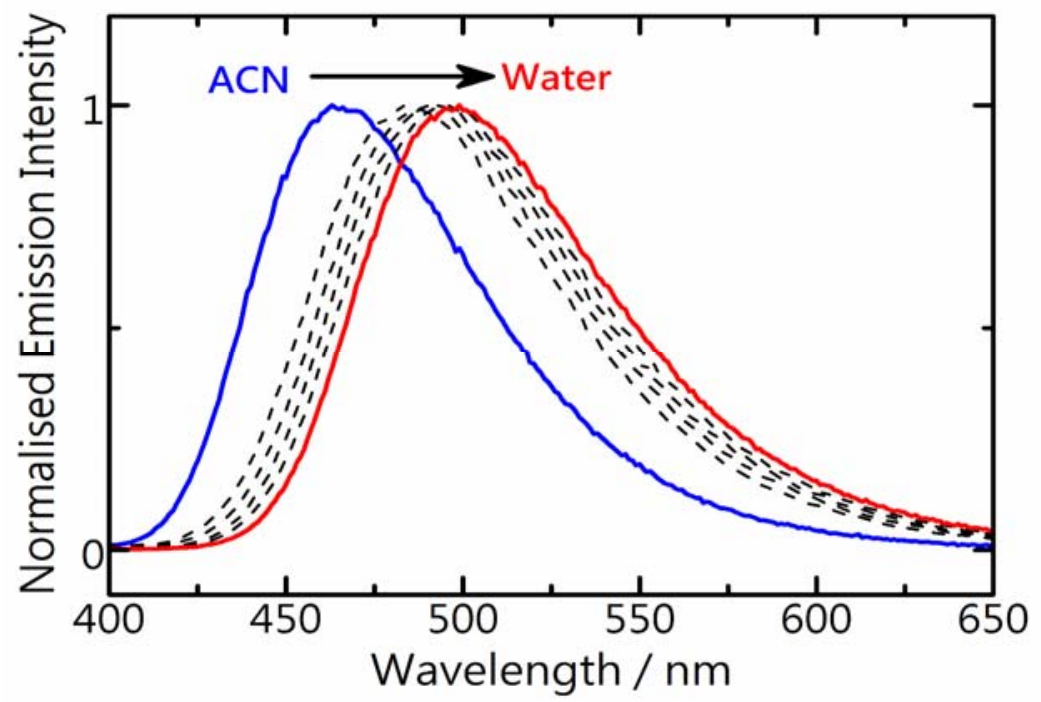

Figure S4. Fluorescence emission spectra of coumarin151 $\left(\lambda_{\mathrm{ex}}=350 \mathrm{~nm}\right)$ in different 8:2, 6:4, 4:6, 2:8 ACN-water mixtures (dashed lines). Emission spectra of coumarin151 in pure $\mathrm{ACN}$ (blue) and in pure water (red) are also appended for easy comparison. 

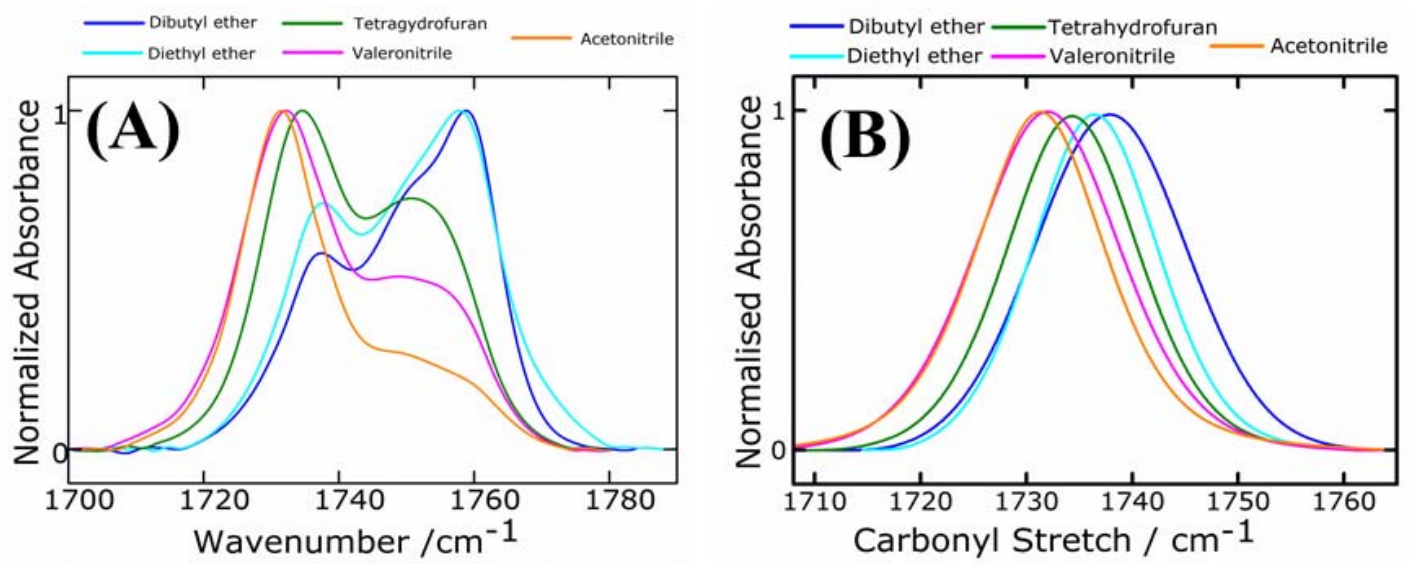

Figure S5. IR absorption spectra of the $\mathrm{C}=\mathrm{O}$ stretch of coumarin151 in polar aprotic solvation environments. (A) Represents the carbonyl stretch of coumarin151 with Fermi resonance and (B) represents the fitted carbonyl stretching frequency of coumarin 151.
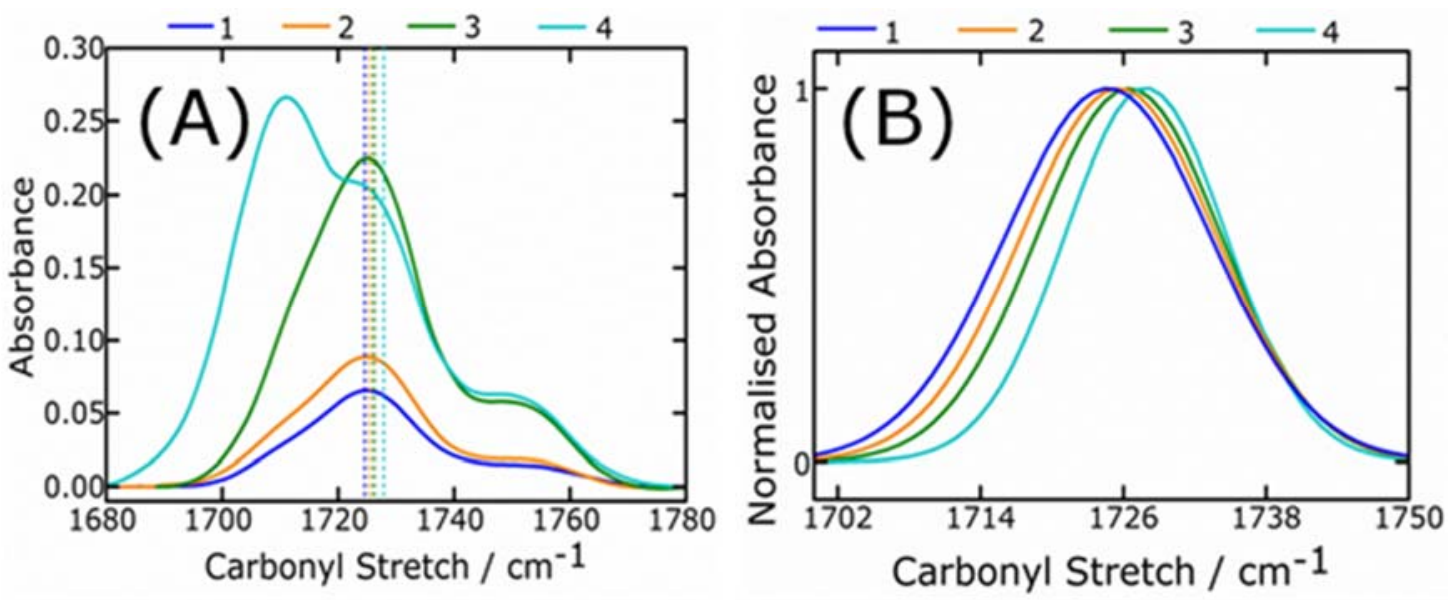

Figure S6. (A) Raw IR absorption spectra of the $\mathrm{C}=\mathrm{O}$ stretch of coumarin 151 in deep eutectic solvents. The numbers $(\mathbf{1}, \mathbf{2}, \mathbf{3}$, and $\mathbf{4})$ correspond to those in Scheme 1. Dotted lines indicate fitted peak position of each IR spectra of the same colour. (B) represents the fitted carbonyl stretching frequency of coumarin151 in DES. 

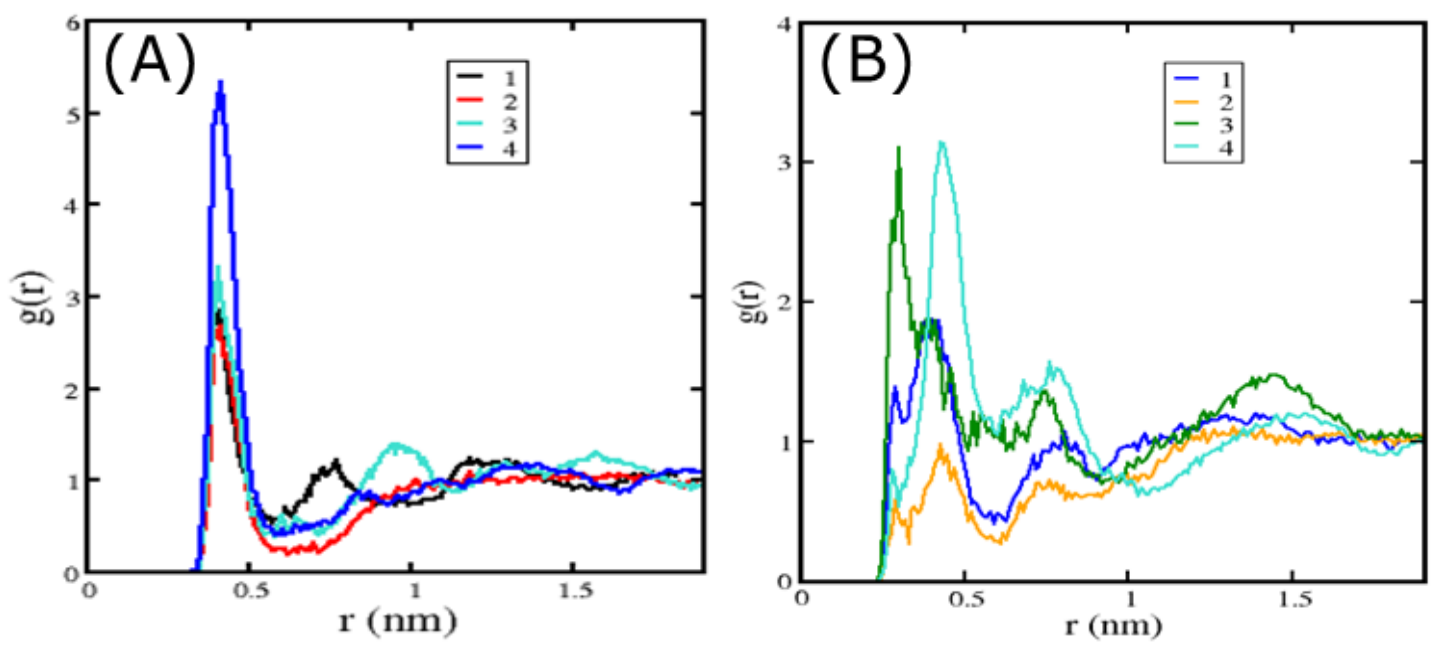

Figure S7. Radial distribution functions (site-site) between coumarin151 carbonyl oxygen and (A) choline nitrogen and (B) choline oxygen.

Tables:

Table S1. Carbonyl IR frequency $\left(\mathrm{cm}^{-1}\right)$, emission maxima $\left(\mathrm{cm}^{-1}\right)$ of coumarin 151 along with predicted electrostatic field from IR frequency and estimated electrostatic field from MD of different DES.

\begin{tabular}{|c|c|c|c|c|l|}
\hline DES & $\begin{array}{c}\text { IR } \\
\text { frequency } \\
/ \mathbf{c m}^{-1}\end{array}$ & $\begin{array}{c}\text { Emission } \\
\text { Maxima } / \mathbf{c m}^{-\mathbf{1}}\end{array}$ & $\begin{array}{c}\text { IR Predicted } \\
\text { Electrostatic } \\
\text { Field }(\mathbf{M V} / \mathbf{c m})\end{array}$ & $\begin{array}{c}\text { MD Estimated } \\
\text { Electrostatic Field } \\
\mathbf{( M V / c m )}\end{array}$ & $\begin{array}{l}\text { Water } \\
\text { Conte } \\
\mathbf{n t} \\
\mathbf{( w t \% )}\end{array}$ \\
\hline $\mathbf{1}$ & 1724.6 & 20387.3 & -47.93 & -47.49 & 0.22 \\
\hline $\mathbf{2}$ & 1725.5 & 20445.7 & -45.05 & -45.72 & 0.13 \\
\hline $\mathbf{3}$ & 1726.2 & 20589.8 & -41.94 & -42.12 & 0.14 \\
\hline $\mathbf{4}$ & 1727.9 & 20780.3 & -37.38 & -38.89 & 0.17 \\
\hline
\end{tabular}

\begin{tabular}{|c|c|c|c|c|c|}
\hline \multicolumn{6}{|c|}{ Table S2. Deconvolution of total electric field to reside specific contribution } \\
\hline \multirow{2}{*}{ DES } & \multicolumn{5}{|c|}{ Electrostatic Field (MV/cm) } \\
\cline { 2 - 6 } & Total & $\begin{array}{c}\text { Chloine } \\
\text { Chloride }\end{array}$ & Alcohol & Chloine cation & $\begin{array}{c}\text { Chloride } \\
\text { anion }\end{array}$ \\
\hline $\mathbf{1}$ & -47.49 & -27.91 & -19.58 & -26.42 & -1.49 \\
\hline $\mathbf{2}$ & -45.72 & -33.04 & -7.86 & -37.86 & 4.82 \\
\hline $\mathbf{3}$ & -42.12 & -32.47 & -8.20 & -40.77 & 8.30 \\
\hline $\mathbf{4}$ & -38.89 & -30.41 & -8.36 & -54.85 & 24.44 \\
\hline
\end{tabular}




\begin{tabular}{|c|c|c|}
\hline DES & $\begin{array}{l}\text { Number of hydrogen bonds from } \\
\text { ChCl }\end{array}$ & $\begin{array}{c}\text { Number of hydrogen bonds from } \\
\text { Alcohol }\end{array}$ \\
\hline 1 & 533 & 1823 \\
\hline 2 & 155 & 1647 \\
\hline 3 & 586 & 1579 \\
\hline 4 & 160 & 702 \\
\hline
\end{tabular}

Table S4. Emission maxima $\left(\mathrm{cm}^{-1}\right)$ of coumarin151 along with predicted electrostatic field from the emission maxima and estimated electrostatic field from MD of different DES.

\begin{tabular}{|c|c|c|c|}
\hline DES & Emission Maxima $/ \mathbf{c m}^{-1}$ & $\begin{array}{c}\text { IR Predicted } \\
\text { Electrostatic Field } \\
\text { (MV/cm) }\end{array}$ & $\begin{array}{c}\text { MD Estimated } \\
\text { Electrostatic Field } \\
\text { (MV/cm) }\end{array}$ \\
\hline $\mathbf{5}$ & 20661.2 & -40.80 & -45.45 \\
\hline $\mathbf{6}$ & 20622.8 & -41.80 & -42.98 \\
\hline $\mathbf{7}$ & 20312.8 & -49.09 & -45.75 \\
\hline
\end{tabular}

